\title{
Effects of phacoemulsification and intraocular lens implantation combined with ciliarotomy in the treatment of angle-closure glaucoma with cataract
}

\author{
BO LI ${ }^{1,2}$, FENG-YUN WANG ${ }^{2}$, TAI-LIANG $\mathrm{LV}^{3}$ and $\mathrm{YU} \mathrm{ZHU}^{1}$
}

\begin{abstract}
${ }^{1}$ Department of Ophthalmology, The First Affiliated Hospital of Zhengzhou University, Zhengzhou, Henan 450052; ${ }^{2}$ Department of Ophthalmology, The First Affiliated Hospital, and College of Clinical Medicine of Henan University of Science Technology, Luoyang, Henan 471003; ${ }^{3}$ Department of Ophthalmology, The Second Affiliated Hospital of Shandong University of Traditional Chinese Medicine, Jinan, Shandong 250001, P.R. China
\end{abstract}

Received February 26, 2016; Accepted June 29, 2016

DOI: $10.3892 / \mathrm{etm} .2016 .3634$

\begin{abstract}
The aim of the present study was to examine the effects of phacoemulsification and intraocular lens implantation combined with ciliarotomy in the treatment of angle-closure glaucoma with cataract in the elderly. A total of 68 patients were consecutively selected and divided into the control group with 33 cases (48 eyes) and the observation group with 35 cases (53 eyes). Cataract surgery combined with trabeculectomy was performed on the patients in the control group and phacoemulsification cataract extraction combined with ciliarotomy was performed on the subjects in the observation group, to compare postoperative effects and complications. Following surgery, the visual acuity of patients in the two groups significantly improved, intraocular pressure decreased, and improvement of the observation group was more evident $(\mathrm{P}<0.05)$. Following surgery, the depth of central anterior chamber and width of chamber angle of patients in two groups was increased, and improvement of the observation group was significantly more evident $(\mathrm{P}<0.05)$. Additionally, the incidence of complications, including corneal swelling, shallow of anterior chamber, fibrinous exudate in iris, and filtering bleb leaking and following cataract removal, of patients in the observation group was significantly reduced compared to the control group $(\mathrm{P}<0.05)$. In summary, the results of the present study show that, phacoemulsification and intraocular lens implantation combined with ciliarotomy in the treatment of angle-closure glaucoma with cataract in the elderly is a safe and effective method and should be applied in the clinic.
\end{abstract}

Correspondence to: Dr Yu Zhu, Department of Ophthalmology, The First Affiliated Hospital of Zhengzhou University, 1 Jianshe Road, Zhengzhou, Henan 450052, P.R. China

E-mail: vkvpvp2994@163.com

Key words: pharmacoemulsification and intraocular lens implantation, ciliarotomy, angle-closure glaucoma, cataract

\section{Introduction}

Primary angle-closure glaucoma is a pathological condition of increased intraocular pressure caused by blockage of aqueous humor flow or angle closure, and constitutes approximately $70 \%$ of glaucoma (1). There is high morbidity in elderly individuals suffering from angle-closure glaucoma (2). Most of their eye structures have degenerative changes, thus the incidence of angle-closure glaucoma with cataract is high at $30 \%$, and has become a common cause of eye diseases resulting in blindness (3).

Simple treatment of glaucoma or cataract surgery does not relieve clinical symptoms effectively, and even increases the risk of recurrence (4). An effective response rate of conventional cataract extracapsular extraction combined with glaucoma operation can be $\leq 50-70 \%$ (5). Phacoemulsification cataract surgery is a possible technique that can be applied in the treatment of cataract combined with glaucoma.

Randomized controlled experiments were carried out in the present study to determine the safety and effectiveness of this type of surgery in the clinic and suggest possible applications thereof.

\section{Materials and methods}

General materials. In total, 68 patients with primary angleclosure glaucoma with cataract presenting at the First Affiliated Hospital of Zhengzhou University were selected between February, 2012 and February, 2014 for the present study. The patients were examined using a non-contact tonometer (Keeler Ltd., London, UK), slit lamp microscope, gonioscopy and ophthalmologic A/B ultrasound machine (all from Shanghai Yimeng Software Technology Co., Ltd., Shanghai, China).

The inclusion criteria for the study were: $\geq 18$ and $<80$ years of age, compliance with the diagnostic code of angle-closure glaucoma suggested by Johnson and Foster (6) and the Emery-Little classification diagnostic code, the range of angle closure from 120 to $270^{\circ}$, patients being administered $>2$ types of drugs to control glaucoma, and progression or intolerance of drugs following the initial treatment. Exclusion criteria for the 
study included the 'iris frill' or collarette syndrome, trauma, tumors and inflammation; medical history of anti-glaucoma operations such as laser, trabeculectomy and iridotomy; medical history of retinal diseases and lens injury, secondary glaucoma such as uveitis and after trauma; and poor compliance and incomplete clinical information.

The patients were randomly divided into the control group with 33 cases (48 eyes) and the observation group with 35 cases (53 eyes). There were 18 male and 15 female patients, aged 47-78 years, with an average of $63.5 \pm 7.1$ years, and angle closure of $130-260^{\circ}$, with an average of $220.4 \pm 23.5^{\circ}$. Regarding hardness level, 16 eyes were lens nucleus with hardness level II, 30 eyes were with level III and 4 eyes were with level IV. The gender, age, range of angle closure and level of lens nucleus hardness in the two groups were compared and there was no statistical difference $(\mathrm{P}>0.05)$.

The present study was approved by the ethics committee of the First Affiliated Hospital of Zhengzhou University. Writtern informed consent was obtained from the patients and their relatives.

Operative methods. Prior to surgery, the patients in the two groups were administered drugs to reduce the intraocular pressure systemically or locally, and intraocular pressure was regulated to $15-30 \mathrm{mmHg}$. The patients received carbonic anhydrase inhibitor orally and used gatifloxacin eye drops. Usage of miotic ceased $24 \mathrm{~h}$ prior to surgery, Mydrin-P ( $0.5 \%$ tropicamide and $0.5 \%$ neophryn) eye drops were used for mydriasis, and the pupil was dilated to $6-7 \mathrm{~mm}$.

Patients in the observation group received combined surgery of glaucoma and cataract. Benoxil superficial anesthesia $(4 \mathrm{~g} / \mathrm{l})$ was combined with Alcaine retrobulbar block anesthesia, a transparent lateral incision was made at the 3 -point corneal limbus of eyes and carbachol miosis was injected to the anterior chamber from it. Subsequently, continuous curvilinear capsularhexis was carried out for $\sim 6 \mathrm{~mm}$. After removing the lens and implanting the intraocular lens, carbachol miosis was injected into the anterior chamber. The corneal limbus was used as the basement to form the scleral flap, resect trabecular meshwork and peripheral iridoplasty in the scleral flap below. Subsequently, micro-nylon was used to suture the scleral and conjunctival flaps intermittently and to seal the ocular region.

Patients in the observation group received phacoemulsification cataract extraction combined with ciliarotomy. On the basis of the level of lens nucleus hardness, appropriate energy was applied in phacoemulsification to remove the lens, and an intraocular lens was implanted in the capsular bag. A viscoelastic agent was injected into the root of the iris in the anterior chamber, and a polishing tool was used to press the root of the iris gently, promoting the anterior angle dissection. After dissection, the viscoelastic agent was removed and the anterior chamber was restored to adjust the position of the intraocular lens and depth of anterior chamber. Following surgery, water was used to close the corneal incision and then seal the ocular region. Following surgery, periocular injection was given with 20,000 units of tobramycin and $2.5 \mathrm{mg}$ of dexamethasone. The operative eyes were wrapped for 3 days continuously after tobradex ointment was applied in the eyes. In between the tobradex ointment, eyedrops were given. The patients with severe reactions in the anterior chamber also received tropicamide for mydriasis. Eventually, the operative eyes with deep anterior chamber and without apophysis of filter bleb were used from the first day following surgery, and were given eye ball massage.

Observation indexes. Vision, intraocular pressure, depth of central anterior chamber, width of chamber angle and postoperative complication rate were compared. Subsequently, an international standard eye chart was used to carry out an eye and vision examination. A tonometer and ophthalmic A ultrasound were employed to detect intraocular pressure and depth of central anterior chamber, respectively, while a microgonioscope (Shanghai Yimeng Software Technology Co., Ltd.) was used to detect the width of the chamber angle.

Statistical analysis. SPSS 20.0 software (IBM SPSS, Armonk, NY, USA) was used for data analysis. Measurement data were presented as mean \pm standard deviation. The independent sample t-test was used for comparisons between groups, and the paired t-test was used for the internal comparison of each group. The number of cases or percentage was used to indicate countable data. The $\chi^{2}$ test was used for comparisons between groups. $\mathrm{P}<0.05$ was considered to indicate a statistically significant difference.

\section{Results}

Comparison of vision and intraocular pressure. The vision and intraocular pressure of patients in the two groups were compared, and no statistical differences were observed $(\mathrm{P}>0.05)$. Following surgery, vision was significantly improved, intraocular pressure was significantly decreased and improvement of the observation group was more evident $(\mathrm{P}<0.05$; Table I $)$.

Comparison of depth of central anterior chamber and width of chamber angle. The difference of depth in the central anterior chamber and width of chamber angle prior to surgery showed no statistical significance $(\mathrm{P}>0.05)$. Following surgery, the depth of the central anterior chamber and width of chamber angle of patients in the two groups was significantly increased, and improvement of the observation group was more evident $(\mathrm{P}<0.05$; Table II).

Comparison of operative complications. The operative complication rate in the observation group was significantly lower than that in the control group $(\mathrm{P}<0.05$; Table III).

\section{Discussion}

Pupil blockage is the main nosogenesis of angle-closure glaucoma. The degeneration of lens structure is the main factor for pupil blockage. Changes in the anatomic structure of local eyeball including thickening of lens, diminution of cornea, shortening of ocular axis and other changes may lead to the occurrence of angle-closure glaucoma (7). Clinical treatment of glaucoma combined with cataract mainly depends on extracting the lens and relieving pupil blockage. Traditional treatment mainly depends on filtrable operation and peripheral 
Table I. Comparison of vision and intraocular pressure.

\begin{tabular}{|c|c|c|c|c|c|c|c|c|}
\hline \multirow[b]{2}{*}{ Group } & \multicolumn{4}{|c|}{ Vision } & \multicolumn{4}{|c|}{ Intraocular pressure, $\mathrm{mmHg}$} \\
\hline & Before operation & After operation & $\mathrm{t}$ & P-value & Before operation & After operation & $\mathrm{t}$ & P-value \\
\hline Observation & $0.12 \pm 0.03$ & $0.67 \pm 0.14$ & 6.032 & 0.025 & $43.25 \pm 5.43$ & $17.56 \pm 3.15$ & 5.327 & 0.035 \\
\hline Control & $0.14 \pm 0.02$ & $0.43 \pm 0.08$ & 4.632 & 0.037 & $41.62 \pm 4.21$ & $26.78 \pm 4.29$ & 4.798 & 0.039 \\
\hline $\mathrm{t}$ & 0.824 & 5.854 & & & 0.914 & 4.969 & & \\
\hline P-value & 0.632 & 0.032 & & & 0.826 & 0.037 & & \\
\hline
\end{tabular}

Table II. Comparison of the depth of central anterior chamber and width of chamber angle.

\begin{tabular}{|c|c|c|c|c|c|c|c|c|}
\hline \multirow[b]{2}{*}{ Group } & \multicolumn{4}{|c|}{ Depth of central anterior chamber, $\mathrm{mm}$} & \multicolumn{4}{|c|}{ Width of chamber angle, $\mathrm{mm}$} \\
\hline & Before operation & After operation & $\mathrm{t}$ & P-value & Before operation & After operation & $\mathrm{t}$ & P-value \\
\hline Observation & $1.21 \pm 0.06$ & $3.74 \pm 0.32$ & 4.698 & 0.037 & $1.24 \pm 0.23$ & $3.97 \pm 0.46$ & 4.967 & 0.036 \\
\hline Control & $1.23 \pm 0.04$ & $2.92 \pm 0.46$ & 3.936 & 0.043 & $1.27 \pm 0.31$ & $3.51 \pm 0.33$ & 4.201 & 0.042 \\
\hline $\mathrm{t}$ & 0.914 & 4.314 & & & 0.745 & 4.426 & & \\
\hline P-value & 0.865 & 0.041 & & & 0.439 & 0.039 & & \\
\hline
\end{tabular}

Table III. Comparisons of operative complications [cases (\%)].

\begin{tabular}{lccccccc}
\hline Group & No. of eyes & $\begin{array}{c}\text { Corneal } \\
\text { swelling }\end{array}$ & $\begin{array}{c}\text { Shallow of } \\
\text { anterior chamber }\end{array}$ & $\begin{array}{c}\text { Exudation of } \\
\text { fibrinoid in iris }\end{array}$ & $\begin{array}{c}\text { Filtering bleb } \\
\text { leaking }\end{array}$ & After-cataract & $\begin{array}{c}\text { Total incidence } \\
\text { rate }\end{array}$ \\
\hline $\begin{array}{l}\text { Observation } \\
\text { Control }\end{array}$ & 53 & 1 & 1 & 1 & 1 & 1 & $5(9.43)$ \\
$\chi^{2}$ & 48 & 3 & 3 & 2 & 2 & 2 & $12(25.0)$ \\
P-value & & & & & & 4.360 \\
\end{tabular}

iridectomy. Filtrable operation cannot resolve shallow of anterior chamber and narrow angle of angle-closure glaucoma fundamentally (8). Due to stimulation of surgical instruments and changes of local metabolism environment following surgery, the lens may experience turbid phenomenon again (9), which increases the rate of a secondary operation, the mental stress and economic pressure of patients.

The development of the phacoemulsification technique and promotion of medical instruments have led to use of phacoemulsification cataract combined with ciliarotomy in the treatment of angle-closure glaucoma combined with cataract, a method that has great application prospect in clinic. The results of the study show that, following surgery, visual acuity in the observation group improved significantly. Intraocular pressure was decreased significantly, the depth of central anterior chamber and width of chamber angle were increased significantly, while operative complications decreased significantly. However, substantial reduction of intraocular pressure prior to surgery to relieve postoperative response and reduce complications (10) is imperative. In addition, ciliarotomy was carried out after completion of cataract extraction because space of the posterior chamber of the eyes is enlarged and the iris became flat after extraction of the lens, since during the process of separating chamber angle, the iris is easy to remove. Ciliarotomy is assessed prior to and after emulsification, and it was found that the differences of vision, intraocular pressure, depth of anterior chamber and opening degree of chamber angle were not evident (11). Mitosis was completed prior to separating the chamber angle in order to increase the tension of iris, which is useful to separate chamber angle and prevents synechia at the same time (12). Before cataract extraction, pupil adhesion was relieved fully, a viscoelastic agent was used or the pupil was gradually dilated, which can reduce damage of the iris sphincter. Curvilinear capsularhexis was applied, a capsule membrane scissors were used to cut off part of the adhered organizational membrane and separate the capsule and cortex was adhered completely in the process of hydration separation (13). An auxiliary hook was used to open the lower iris when phacoemulsification lens cleaved the nucleus, in situ phacoemulsification was assessed directly, and the nucleus was divided into two sections using the stop-and-chop method to divide the remaining nucleus into 2-3 fragmentation nucleus, which was successively removed while avoiding placing the probe of ultrasonic emulsification into the iris to operate blindly (14). The auxiliary hook was used to avoid the iris while perfusing the cortex, polishing 
the posterior capsule and implanting the foldable intraocular lens into a capsular bag. After separating the chamber angle with a viscoelastic agent, it was completely removed from the anterior chamber, and pigments and organizations. The remaining viscoelastic agent in the intraocuar lens was also removed, as well as caducous iris pigments, white organizational membranes and other residues in the surface and capsular bag (15).

In summary, use of phacoemulsification and intraocular lens implantation combined with ciliarotomy to treat angle-closure glaucoma with cataract in the elderly is a safe and effective technique, and should be applied in the clinic.

\section{References}

1. Liu CJ, Cheng CY, Ko YC and Lau LI: Determinants of long-term intraocular pressure after phacoemulsification in primary angle-closure glaucoma. J Glaucoma 20: 566-570, 2011.

2. Liang YB, Wang NL, Rong SS and Thomas R: Initial treatment for primary angle-closure glaucoma in China. J Glaucoma 24: 469-473, 2015.

3. Guo JM, Li M, Xu XL, Zhang H and Wang JM: Anterior segment changes after pharmacologic mydriasis using Pentacam and optical coherence tomography in angle closure suspects. Int J Ophthalmol 8: 980-984, 2015.

4. Verma J, John D, Nair SR, Oomman S, Mishra R, Shah P, Jha D and Shaikh S: Efficacy and safety of surgical treatment options for primary angle closure glaucoma: A meta-analysis of randomised controlled trials. Value Health 18: A415-A416, 2015.

5. Marchini G, Chemello F, Berzaghi D and Zampieri A: New findings in the diagnosis and treatment of primary angle-closure glaucoma. Prog Brain Res 221: 191-212, 2015.

6. Johnson GJ and Foster PJ: Can we prevent angle-closureglaucoma? Eye (Lond) 19: 1119-1124, 2005.
7. Nongpiur ME, Png O, Chiew JW, Fan KR, Girard MJ, Wong T, Goh D, Perera SA and Aung T: Lack of association between corneal hysteresis and corneal resistance factor with glaucoma severity in primary angle closure glaucoma. Invest Ophthalmol Vis Sci 56: 6879-6885, 2015.

8. Zetterberg M: Age-related eye disease and gender. Maturitas 83: 19-26, 2016.

9. Waisbourd M, Savant SV, Sun Y, Martinez P and Myers JS: Water-drinking test in primary angle-closure suspect before and after laser peripheral iridotomy. Clin Experiment Ophthalmol 44: 89-94, 2016.

10. Goel N, Sharma R, Sawhney A, Mandal M and Choudhry RM: Lensectomy, vitrectomy, and transvitreal ciliary body photocoagulation as primary treatment for glaucoma in microspherophakia. J AAPOS 19: 366-368, 2015.

11. Sng CC, Aquino MC, Liao J, Zheng C, Ang M and Chew PT: Anterior segment morphology after acute primary angle closure treatment: a randomised study comparing iridoplasty and medical therapy. Br J Ophthalmol 100: 542-548, 2016.

12. Dada T, Rathi A, Angmo D, Agarwal T, Vanathi M, Khokhar SK and Vajpayee RB: Clinical outcomes of clear lens extraction in eyes with primary angle closure. J Cataract Refract Surg 41: 1470-1477, 2015

13. Hou X, Hu D, Cui Z, Zhou J, Cai L and Wang Y: Small-incision phacotrabeculectomy versus phacoemulsification in refractory acute primary angle closure with cataract. BMC Ophthalmol 15: $88,2015$.

14. Day AC, Burr JM, Bunce C, Doré CJ, Sylvestre Y, Wormald RP, Round J, McCudden V, Rubin G and Wilkins MR; FACT Group: Randomised, single-masked non-inferiority trial of femtosecond laser-assisted versus manual phacoemulsification cataract surgery for adults with visually significant cataract: The FACT trial protocol. BMJ Open 5: e010381, 2015.

15. Lundström M, Manning S, Barry P, Stenevi U, Henry Y and Rosen P: The European registry of quality outcomes for cataract and refractive surgery (EUREQUO): A database study of trends in volumes, surgical techniques and outcomes of refractive surgery. Eye Vis Lond 2: 8, 2015. 